E.M. Palacios, MSc

R. Sala-Llonch, MSc

C. Junque, $\mathrm{PhD}$

T. Roig, $\mathrm{PhD}$

J.M. Tormos, MD, PhD

N. Bargallo, MD, PhD

P. Vendrell, PhD

Correspondence \& reprint requests to Dr. Junque: cjunque@ub.edu
Editorial, page $\mathrm{XXX}$

Supplemental data at www.neurology.org

\section{White matter integrity related to functional working memory networks in traumatic brain injury}

\section{ABSTRACT}

Objective: This study explores the functional and structural patterns of connectivity underlying working memory impairment after severe traumatic axonal injury.

Methods: We performed an fMRI n-back task and acquired diffusion tensor images (DTI) in a group of 19 chronic-stage patients with severe traumatic brain injury (TBI) and evidence of traumatic axonal injury and 19 matched healthy controls. We performed image analyses with FSL software and $\mathrm{fMRI}$ data were analyzed using probabilistic independent component analysis. Fractional anisotropy (FA) maps from DTI images were analyzed with FMRIB's Diffusion Toolbox.

Results: We identified working memory and default mode networks. Global FA values correlated with both networks and FA whole-brain analysis revealed correlations in several tracts associated with the functional activation. Furthermore, working memory performance in the patient group correlated with the functional activation patterns and with the FA values of the associative fasciculi.

Conclusion: Combining structural and functional neuroimaging data, we were able to describe structural white matter changes related to functional network alterations and to lower performance in working memory in chronic TBI. Neurology ${ }^{\circledR}$ 2012;78:1-1

\section{GLOSSARY}

BOLD = blood oxygenation level-dependent; $\mathbf{D T I}=$ diffusion tensor image; $\mathbf{E P I}=$ echoplanar imaging; $\mathbf{F A}=$ fractional anisotropy; FOV = field of view; FWE = family-wise error; IC = independent component; ICA = independent component analysis; MNI = Montreal Neurological Institute; TBI = traumatic brain injury; TE = echo time; TFCE = threshold-free cluster enhancement; $\mathbf{T I}=$ inversion time; $\mathbf{T R}=$ repetition time

Multimodal integration studies of brain connectivity allow the investigation of the relationship between brain structure and function. Areas with strongly correlated activity are more likely to be anatomically connected ${ }^{1}$ although increased functional connectivity does not necessarily predict increased structural connectivity. ${ }^{2}$ Traumatic axonal injury causes multifocal damage, disrupting critical cortical-subcortical circuitry leading to significant functional consequences, and cognitive dysfunction after traumatic brain injury (TBI). 3,4 Working memory is frequently impaired following TBI. fMRI studies reported patterns of increased or decreased cerebral activations in the regions that form part of the working memory network, ${ }^{5-8}$ and white matter alterations measured by diffusion tensor imaging (DTI) correlated with working memory impairment in severe TBI. ${ }^{9}$

However, no previous studies with TBI patients have combined these techniques to investigate the relationship between the functional and structural substrates underlying working memory impairment. In this study, we assessed white matter integrity and functional brain connectivity using DTI and independent component analyses (ICA), respectively. The ICA method extracts patterns or independent components (IC) that reflect brain functional connectivity. Each IC consists of a spatial map that shows a pattern of synchronized activation over time of different brain regions in response to stimuli. ${ }^{10,11}$ We hypothesized that 1 ) white matter alteration would affect functional

From the Department of Psychiatry and Clinical Psychobiology (E.M.P., R.S.-L., C.J., P.V.), University of Barcelona, Barcelona; Institute of Biomedical Research August Pi i Sunyer (IDIBAPS) (E.M.P., R.S.-L., C.J., P.V.), Barcelona; Institut Universitari de Neurorehabilitacio Guttmann (T.R., J.M.T.), Badalona; and Centre de Diagnostic per la Imatge Hospital Clinic de Barcelona (CDIC) (N.B.), Hospital Clinic de Barcelona, Spain. Study funding: Sponsored by the MAPFRE Foundation.

Disclosure: Author disclosures are provided at the end of the article. 
working memory and default mode networks; and 2) alterations in both white matter integrity and functional networks would explain working memory deficits.

METHODS Subjects. We performed a cross-sectional study of 38 subjects. We recruited 19 patients with TBI from a data-

\begin{tabular}{|c|c|c|c|}
\hline Table 1 & \multicolumn{3}{|c|}{ Clinical and neuroimaging characteristics of the TBI patients } \\
\hline Patient & Tev & GCS & MRI findings (T2*/FLAIR-hemosiderin deposits): MBs/contusions \\
\hline 1 & 6 & 3 & $\begin{array}{l}\text { MBs in } R / L \text { thalamus, internal capsule, midbrain, } L \text { hippocampus, } \\
\mathrm{L} \text { temporal, frontal lobe and genu CC. Parahippocampal } \\
\text { contusion }(0.9 \mathrm{~mL}) \text {. }\end{array}$ \\
\hline 2 & 5.9 & 6 & $\begin{array}{l}\text { MBs in } R \text { thalamus, } L \text { caudate, and frontotemporal lobes. Frontal } \\
\text { and CC deep white matter hyperintensities due to demyelination. } \\
\text { Frontal contusion }(0.7 \mathrm{~mL}) \text {. }\end{array}$ \\
\hline 3 & 5.7 & 3 & $\begin{array}{l}\text { MBs in } R \text { caudate, } R \text { internal capsule, } R \text { temporal lobe, } R \text { lentiform } \\
\text { nucleus, } R / L \text { frontoparietal lobes, } R \text { occipital lobe, and splenium CC. } \\
\text { Frontoparietal deep white matter hyperintensities due to } \\
\text { demyelination. No contusions evidenced. }\end{array}$ \\
\hline 4 & 3.4 & 7 & $\begin{array}{l}\text { MBs in R hippocampus, splenium CC, R cerebellum, } R \text { frontal } \\
\text { hemorrhagic contusion ( } 4.8 \mathrm{~mL}) \text {. }\end{array}$ \\
\hline 5 & 5.6 & 4 & $\begin{array}{l}\text { MBs in } L \text { thalamus, } R \text { insula, } R / L \text { hippocampus, } R / L \text { frontal lobes, } \\
\text { and splenium CC. Frontal deep white matter hyperintensities } \\
\text { due to demyelination. No contusions evidenced. }\end{array}$ \\
\hline 6 & 3.4 & 8 & $\begin{array}{l}\text { MBs in CC and frontoparietal lobes bilaterally. Periventricular } \\
\text { and frontoparietal deep white matter affectation due to } \\
\text { demyelinating or malacic myelopathy. No contusions evidenced. }\end{array}$ \\
\hline 7 & 2.6 & 3 & $\begin{array}{l}\text { MBs in } R / L \text { thalamus; } R / L \text { insula, } R / L \text { external capsule, } L \\
\text { lentiform nucleus, } L / R \text { hippocampus, } R / L \text { cortico-subcortical } \\
\text { frontoparietal junction, temporal and occipital lobes, splenium } \\
\text { and body } C C \text {, and cerebellum. No contusions evidenced. }\end{array}$ \\
\hline 8 & 3.8 & 6 & $\begin{array}{l}\text { MBs in } L \text { frontal lobe, } R \text { temporal lobe, midbrain, and cerebellum. } \\
\text { Small } L \text { parietal deep white matter focus of demyelination. } \\
\text { No contusions evidenced. }\end{array}$ \\
\hline 9 & 7.4 & 6 & $\begin{array}{l}\text { MBs in } L \text { thalamus, midbrain, frontotemporal deep white matter } \\
\text { affectation. Temporal contusions (L: } 6.08 \mathrm{~mL}, 8.4 \mathrm{~mL} ; \mathrm{R}: 1.8 \mathrm{~mL} \text {, } \\
1.9 \mathrm{~mL}) \text {, frontal contusion }(9.3 \mathrm{~mL}) \text {. }\end{array}$ \\
\hline 10 & 5 & 5 & $\begin{array}{l}\text { MBs in midbrain, cerebellum, splenium CC. Deep frontal white } \\
\text { matter hyperintensities due to demyelination. No contusions } \\
\text { evidenced. }\end{array}$ \\
\hline 11 & 3.4 & 3 & $\begin{array}{l}\text { MBs in } L \text { insula, basal ganglia, midbrain, } L \text { hippocampus, } \\
\text { frontoparietal lobes. Deep R temporal and parietal white matter } \\
\text { hyperintensities due to demyelination. Frontobasal }(1.07 \mathrm{~mL}) \\
\text { and temporal }(1.3 \mathrm{~mL}) \text { contusions. }\end{array}$ \\
\hline 12 & 2.5 & 4 & $\begin{array}{l}\text { MBs in } L \text { thalamus, R hippocampus, and splenium CC. Deep } \\
\text { frontal white matter hyperintensities. No contusions evidenced. }\end{array}$ \\
\hline 13 & 4.6 & 6 & $\begin{array}{l}\text { MBs R external capsule, pons, cerebellum, and splenium CC. } \\
\text { Deep temporal white matter hyperintensities. Insular contusion } \\
\text { ( } 2.1 \mathrm{~mL} \text { ). }\end{array}$ \\
\hline 14 & 4.5 & 4 & $\begin{array}{l}\text { MBs in } \mathrm{L} \text { caudate, } \mathrm{R} / \mathrm{L} \text { hippocampus, midbrain, frontoparietal } \\
\text { lobes, and splenium and genu CC. }\end{array}$ \\
\hline 15 & 3.3 & 7 & $\begin{array}{l}\text { MBs in } L \text { parietal and temporal lobes, and } R \text { occipital lobe. Deep } \\
\text { periventricular white matter hyperintensities. }\end{array}$ \\
\hline 16 & 2.3 & 8 & $\begin{array}{l}\text { MBs in L lentiform nucleus, midbrain, } R \text { frontoparietal lobes, } R \\
\text { temporal lobe, and splenium CC. No contusions evidenced. }\end{array}$ \\
\hline 17 & 3 & 5 & $\begin{array}{l}\text { MBs in genu CC and parietotemporal lobes. Frontotemporal } \\
\text { MBs related to contusions. Temporal lobe contusions }(4.08 \mathrm{~mL}, \\
12.8 \mathrm{~mL}), \mathrm{R} \text { frontal contusion }(3.9 \mathrm{~mL}), \mathrm{L} \text { frontal contusion }(6.7 \mathrm{~mL}) \text {. }\end{array}$ \\
\hline 18 & 3.9 & 3 & $\begin{array}{l}\text { MBs R/L in frontal and temporal lobes. Deep temporal and } \\
\text { frontal white matter hyperintensities due to demyelination. } \\
\text { No contusions evidenced. }\end{array}$ \\
\hline 19 & 1.8 & 5 & $\begin{array}{l}\text { MBs in R/L frontal lobe. Deep frontal white matter hyperintensities. } \\
\text { No contusions evidenced. }\end{array}$ \\
\hline
\end{tabular}

Abbreviations: $\mathrm{CC}=$ corpus callosum; FLAIR = fluid-attenuated inversion recovery; GCS = Glasgow Coma Scale; MBs = microbleeds; $\mathrm{TBI}=$ traumatic brain injury; $\mathrm{Tev}=$ time of evolution since the accident to the MRI evaluation (years). base of 366 chronic patients at the Head Injury Unit of the Institut de Neurorehabilitació Guttmann. After excluding patients living outside the area of Barcelona $(\mathrm{n}=175)$, we applied the following inclusion criteria: 1) severe closed-head injury and severe TBI defined as Glasgow Coma Scale score $\leq 8 ; 2$ ) adults aged $\leq 40$ years; 3 ) chronic stage of recovery $\geq 2$ years since the TBI; 4) possible diffuse pathology reported in the MRI scans in the subacute stage without macroscopic lesions. The exclusion criteria were 1) visual, sensorial, or visuoperceptive deficits; 2) previous history of TBI, drug intake, neurologic, or psychiatric disorders; 3) injury requiring craniectomy or craniotomy. Sixtyseven patients met these criteria and were phoned consecutively until a sample of 44 participants were obtained, who were then included in a study of the long-term consequences of severe TBI. Since we were interested in diffuse white matter injury after TBI, the neuroradiologist (N.B.) described the chronic brain lesions seen in the current MRI. Patients with large lesions were excluded, similarly to previous studies in the traumatic axonal injury literature. ${ }^{12}$ This left us with 27 subjects. We excluded 3 patients because of motion artifacts in the MRI, and 5 for lack of collaboration. The final patient group comprised 19 patients. Table 1 shows their clinical and neuroimaging characteristics. The etiology of TBI was traffic accident in all cases.

Nineteen healthy volunteers matched by age, sex, education, and handedness were recruited as the control group (table 2). None had a previous history of neurologic or psychiatric diseases and brain scans were reported as normal. In patients, we evaluated handedness by premorbid writing hand preference.

Standard protocol approvals and patient consent. The Research Ethics Committees of the Institut Universitari de Neurorehabilitació Guttmann and the University of Barcelona approved the study. All participants gave written informed consent.

Neuropsychological assessment. To evaluate the current neurocognitive status of the subjects, a trained neuropsychologist blinded to the clinical data administered the tests assessing executive function, verbal and visual memory, visuoperception, and processing speed, all common deficits after TBI. We administered the neuropsychological assessment 1 week before the fMRI in order to identify possible motor, sensorial, or perceptual deficits that might interfere with the fMRI task. The assessment included Letter-Number Sequencing and Digit Span Forward and Backwards test, ${ }^{13}$ Trail-Making Test A and B, ReyOsterrieth complex figure, Rey Auditory Verbal Learning Test, Stroop reading and color-naming conditions, and verbal fluencies. ${ }^{14}$ Results are summarized in table 2.

Image acquisition. The fMRI data were acquired on a Siemens Magnetom TrioTim syngo 3-Tesla at the Centre de Diagnostic per la Imatge of the Hospital Clinic (CDIC), Barcelona. We acquired a high-resolution T1-weighted structural image scan for each subject with a magnetization-prepared rapid gradient echo 3-dimensional protocol (repetition time $[\mathrm{TR}]=2,300$ msec; echo time $[\mathrm{TE}]=3 \mathrm{msec}$; inversion time $[\mathrm{TI}]=900$ msec; field of view $[\mathrm{FOV}]=244 \mathrm{~mm} ; 1 \mathrm{~mm}$ isotropic voxel) and a single shot gradient-echo echoplanar imaging (EPI) sequence $(\mathrm{TR}=2,000 \mathrm{msec} ; \mathrm{TE}=16 \mathrm{msec}$; flip angle $=90$ grad; FOV $=220 \mathrm{~mm}$; voxel size $=1.7 \times 1.7 \times 3.0 \mathrm{~mm}$ ) for fMRI. Visual stimuli were projected on a screen seen through a mirror mounted on the head coil. Diffusion-weighted images were sensitized in 30 noncollinear directions with a b-value $=1,000$ $\mathrm{s} / \mathrm{mm}^{2}$, using an EPI sequence $(\mathrm{TR}=9,300 \mathrm{msec}$; $\mathrm{TE}=94$ msec; slice thickness $=2.0 \mathrm{~mm}$; voxel size $=2.0 \times 2.0 \times 2.0$ 
Table 2 Demographic and neuropsychological characteristics of patients and controls

\begin{tabular}{|c|c|c|c|}
\hline & $\begin{array}{l}\text { TBI group, } \\
\text { mean (SD) }\end{array}$ & $\begin{array}{l}\text { Control group, } \\
\text { mean (SD) }\end{array}$ & $p$ Value \\
\hline Age, $y$ & $26.78(5.55)$ & $27.47(6.04)$ & 0.72 \\
\hline \multicolumn{4}{|l|}{ Education } \\
\hline Elementary school & 3 & 4 & \\
\hline Secondary education & 12 & 10 & \\
\hline College and university & 4 & 4 & 0.86 \\
\hline \multicolumn{4}{|l|}{ Sex } \\
\hline$M$ & 12 & 10 & \\
\hline $\mathbf{F}$ & 7 & 9 & 0.52 \\
\hline \multicolumn{4}{|l|}{ Handedness } \\
\hline $\mathbf{R}$ & 15 & 15 & \\
\hline L & 4 & 3 & 0.73 \\
\hline \multicolumn{4}{|c|}{ Neuropsychological performance } \\
\hline TMT A & $31.68(10.9)$ & $22.72(6.23)$ & 0.05 \\
\hline TMT в & $79.16(22.7)$ & $57.28(14.15)$ & 0.001 \\
\hline TMT A \& B & 47.47 (21.43) & $35.11(16.1)$ & 0.056 \\
\hline Digit forward & $9.37(1.9)$ & $9.90(2.32)$ & 0.46 \\
\hline Digit backward & $6.59(1.12)$ & 7.61 (1.6) & 0.03 \\
\hline Symbol digit & $69.74(13.25)$ & $86(14.4)$ & 0.001 \\
\hline Stroop reading & $98(14.67)$ & $109.61(13.28)$ & 0.01 \\
\hline Stroop color naming & $64.58(8.46)$ & $77.72(9.12)$ & $<0.001$ \\
\hline Stroop interference & $6.86(6.18)$ & $7.62(4.36)$ & 0.66 \\
\hline LNS & $10.37(2.06)$ & $12.50(1.6)$ & 0.001 \\
\hline \multicolumn{4}{|l|}{ Fluencies } \\
\hline Semantic & 22.44 (11.68) & $24(4.85)$ & 0.62 \\
\hline Phonemic & $34.53(12.4)$ & 45.5 (9.4) & 0.005 \\
\hline RAVLT (DR) & $8.32(3.44)$ & $13.56(1.25)$ & $<0.001$ \\
\hline ROCF (DR) & 19.05 (5.9) & $23.44(4.44)$ & 0.015 \\
\hline \multicolumn{4}{|l|}{$\mathrm{N}$-back task } \\
\hline 0-back (RT) & $0.55(0.08)$ & $0.49(0.07)$ & 0.06 \\
\hline 0-back d' & $3.20(1.24)$ & $4.12(0.18)$ & 0.005 \\
\hline 2-back (RT) & $0.59(0.1)$ & $0.54(0.08)$ & 0.03 \\
\hline 2-back d' & $2.47(0.85)$ & $3.32(0.66)$ & 0.001 \\
\hline
\end{tabular}

Abbreviations: $d^{\prime}=$ accuracy measure; DR = delayed recall; LNS $=$ Letter-Number Sequencing (Wechsler Adult Intelligence Scale-III); RAVLT = Rey Auditory Verbal Learning Test; ROCF = Rey-Osterrieth Complex Figure; $\mathrm{RT}$ = reaction time (s); TBI = traumatic brain injury; TMT $=$ Trail-Making Test.

$\mathrm{mm} ; \mathrm{FOV}=240 \mathrm{~mm}$; no gap). Subjects were scanned twice. In the first scan we acquired the structural sequences and in the second scan performed a week later we acquired the fMRI data.

fMRI paradigm. We administered a visual facial ${ }^{15} \mathrm{n}$-back task. The $\mathrm{n}$-back paradigm is widely used to investigate the neural basis of working memory. ${ }^{16}$ The experimental conditions were 0-back (control task) and 2-back (working memory task). In the 0 -back condition, we asked individuals to decide whether the stimuli matched a single target stimulus specified before the epoch began. During the 2-back condition, they were asked to decide whether the stimuli currently presented matched the stimuli that had been presented 2 trials previously. Each n-back condition involved a 30-second epoch, preceded by a 2 -second short written instruction. The design alternated 0 -back and 2-back conditions starting with 0 -back. We used a block design with 16 cycles of alternation between conditions, presented in the course of the 8-minute experiment. There was no period of control fixation between each condition. Each stimulus appeared on the screen for 1 second against a black background, followed by a fixation cross for another 1 second. Participants had to press a button only when the currently presented stimulus was a target. We registered hits, misses, correct rejects, false alarms, and reaction times. Prior to the scan, participants rehearsed a shorter familiarization session of the task, reaching an accuracy plateau of $80 \%$. We recorded reaction times and calculated the $\mathrm{d}^{\prime}$ measure, a bias-free measure that takes both correct answers and errors into account, to determine the accuracy of performance. Higher accuracy measures $\left(\mathrm{d}^{\prime}\right)$ indicate higher performance.

fMRI preprocessing and analysis. For the fMRI data preprocessing we used the following tools of the FSL software (http://www.fmrib.ox.ac.uk/fsl): motion correction, removal of nonbrain structures, spatial smoothing, mean-based intensity normalization, and high-pass temporal filtering. The functional scans were then registered to the MNI152 standard space. We preprocessed the images using probabilistic ICA as implemented in MELODIC. ${ }^{17}$ MELODIC decomposes the fMRI data into a set of ICs, where each IC is composed of a spatial map, a time course, and a vector of subject modes (Smodes) (appendix e-1 on the Neurolog ${ }^{\circledR}$ Web site at www.neurology.org).

DTI preprocessing and analysis. We used FSL Diffusion Toolbox. After calculation of the FA map for each subject, we implemented a voxel-wise statistical analysis of the FA data using Tract-Based Spatial Statistics v1.2. We obtained global FA values from each subject's FA skeleton map (appendix e-1).

Gray matter volume analysis. We applied the SIENAX tool from FSL for estimation of brain gray matter volume (appendix e-1).

Statistics. For the fMRI data, we performed a post hoc regression analysis on the output estimated time series from the tasktemporal series to identify the components related to each task condition. After identification of ICs of interest, data were regressed against 2 kinds of general linear model matrix. First we tested for differences between groups using a $t$ test; we then assayed relationships between global FA values and patterns of functional activation, and then examined whether the activation correlated with task performance. We analyzed control and patient groups separately.

For the DTI data, we performed correlations between whole brain FA maps and mean blood oxygenation level-dependent (BOLD) response within the network for each subject and working memory performance (2-back accuracy measure $\mathrm{d}^{\prime}$ ) using Randomize v2.1 from FSL. ${ }^{18,19}$ The statistical threshold was set at $p<0.05$ family-wise error (FWE) corrected. The threshold-free cluster enhancement (TFCE) method was used to define the clusters. ${ }^{20}$

For statistical analyses of nonimaging data we used SPSS v17 (SPSS Inc., Chicago, IL). We examined group differences with the Student $t$ test and used $p<0.05$ as the threshold of significance.

RESULTS Behavioral functional data. Table 2 summarizes the mean RT and accuracy measure $\left(d^{\prime}\right)$ for TBI patients and healthy controls for the 2 task conditions. TBI differed from controls in both accuracy and RT measures. 
Identification of functional brain activation patterns. Decomposition of the fMRI dataset resulted in 36 different activation patterns of connectivity (ICs). We selected the first 2 components as having the highest $Z$ value and highest correspondence with the temporality of the stimuli, and avoided the ones representing known artifacts such as noise, motion, or venous pulsation.

The first activation pattern, IC1, was associated with the 0 -back $>2$-back contrast $(p<0.001)$ and its spatial activation maps corresponded to the default mode network (figure 1A). The brain regions that presented activations included the precuneus, frontal pole, medial temporal gyrus, cingulate gyrus, temporal fusiform cortex, and bilateral inferior parietal cortex. This component also included deactivations involving the superior frontal gyrus, superior parietal lobe, frontal pole, lingual gyrus, insular cortex, and the paracingulate gyrus.

The second pattern of activation, IC2, was related to the 2-back $>0$-back contrast $(p<0.001)$. The distribution of the topographic activation corresponded to the working memory network (figure 1B). Activated brain regions included the superior parietal lobes, middle frontal gyrus, insular cortex, temporal fusiform gyrus, middle temporal gyrus, and anterior paracingulate gyrus. Deactivations for this condition were observed in the following areas: cingulate gyrus, frontal pole, middle temporal gyrus, bilateral inferior parietal cortex, orbitofrontal cortex, left superior temporal gyrus, and precentral gyrus. Table e-1 summarizes the cerebral areas, Montreal Neurological Institute (MNI) maximum coordinates, and cluster size of the spatial maps.

We found a significant correlation between the activation of the working memory network and the deactivation of the default mode network for both the TBI group $(r=0.753, p<0.001)$ and the control group $(r=0.877, p<0.001)$.

TBI patients showed reduced default mode network activity in group comparison. We found statistically significant differences between groups for the activation pattern IC1. Patients showed decreased activation compared to the control group in the cerebral regions that corresponded to the default mode network $(p<0.009)$. For the working memory activation pattern IC2, we found a trend toward significance, suggesting that patients also had reduced activation, especially in the parietal lobes, in comparison with the control group $(p<0.06)$.

To control for the effects of diffuse white matter damage on brain activation, we selected the pattern corresponding to the visual system. There were no group differences in the activation of this component (patients, mean $=1.76$; controls, mean $=1.24 ; p=0.45$ ).
White matter integrity correlated with functional activity in TBI patients. Global FA measures correlated with the default mode network deactivations and the activations of the working memory network $(r=$ $0.41, p=0.04$ and $r=0.57, p=0.006$ ), respectively. Patients with reduced white matter integrity showed a lower level of activation in these functional networks for both default mode network and working memory.

In TBI patients, but not in controls, FA maps correlated with individual functional activation. $\mathrm{Pa}-$ tients with better white matter integrity had higher activation in both networks. The fasciculi that achieved significant correlations were intrahemispheric association fibers of the inferior and superior longitudinal fasciculi, inferior fronto-occipital fasciculi, and the cingulum bundle; interhemispheric fibers of the corpus callosum (genu and splenium) and projection fibers of the corticospinal tracts as well as in the anterior thalamic radiations, anterior limb of the internal capsule, and anterior corona radiata. Figure 2 illustrates FWE-corrected maps $p<0.05$ (TFCE).

Impairment of specific fasciculi explained working memory performance. FA values correlated with the 2-back accuracy measure in the TBI patients, FWEcorrected $p<0.05$. Patients with better white matter integrity in specific fasciculi performed better in the working memory task. Figure 3 summarizes the fasciculi that achieved significant correlations. We did not find significant correlations for the control group.

Working memory performance correlated with functional and structural measures. We found significant correlations for the TBI patients in the default mode and working memory networks with the accuracy measure $\mathrm{d}^{\prime}(r=0.53, p=0.009)$. These results indicate that patients with better performance had higher activation in the working memory network and increased deactivation of the default mode network.

No correlations were found between the gray matter volume and working memory performance $(r=0.31 ; p=0.10)$.

DISCUSSION We combined fMRI and DTI techniques to obtain a better understanding of the connectivity alterations underlying working memory impairment after severe traumatic axonal injury. We demonstrate that alterations in the patterns of functional activity can be explained by structural connectivity damage in TBI patients. Moreover, these alterations correlated with poorer performance on the working memory fMRI task.

In our study, we found 2 different patterns of activation. One of these patterns corresponded to the 
Figure 1 Default mode and working memory networks identified in the fMRI analysis of the groups
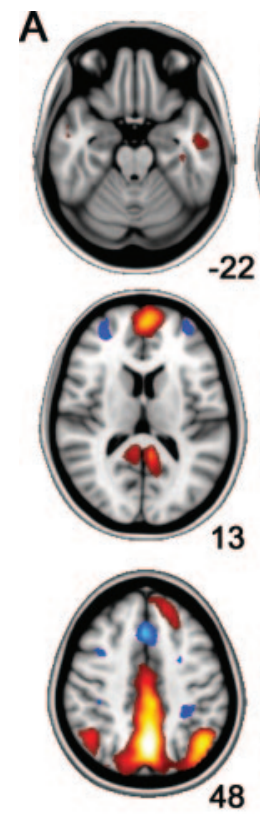

13

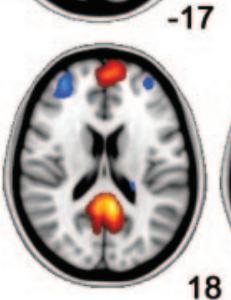

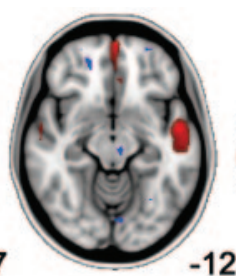

$-12$

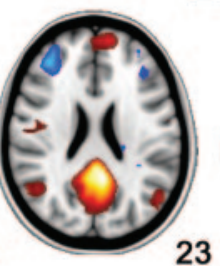

23
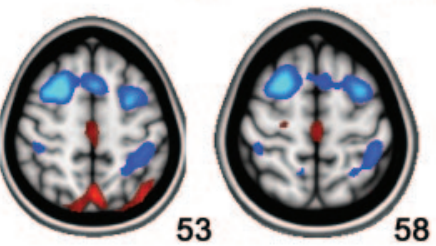
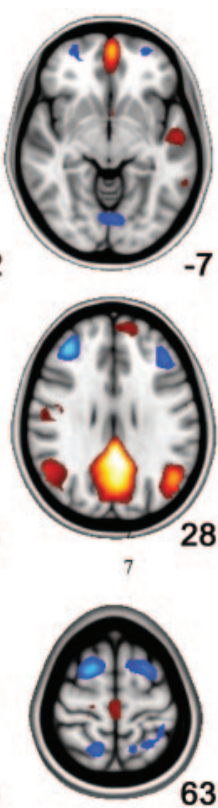
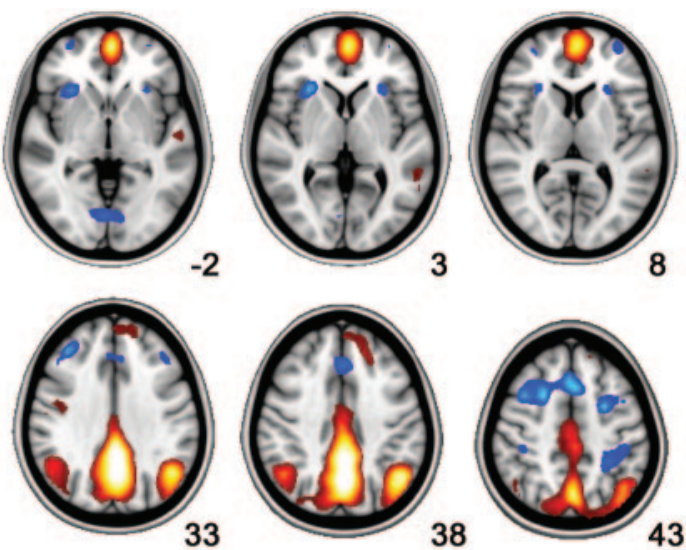

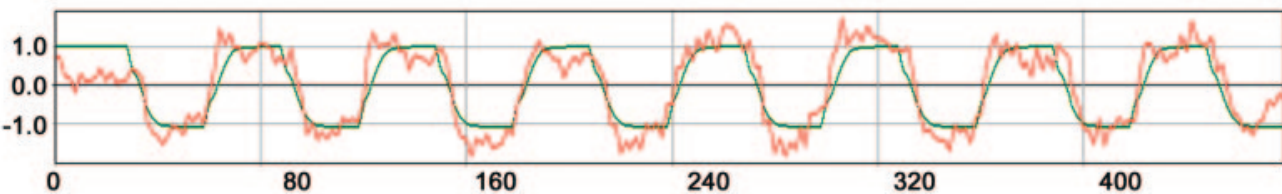

- Time course
- Full model fit
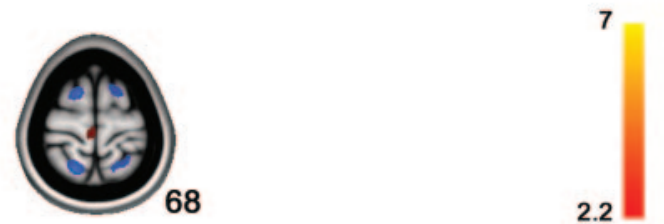

$-2.2$

B
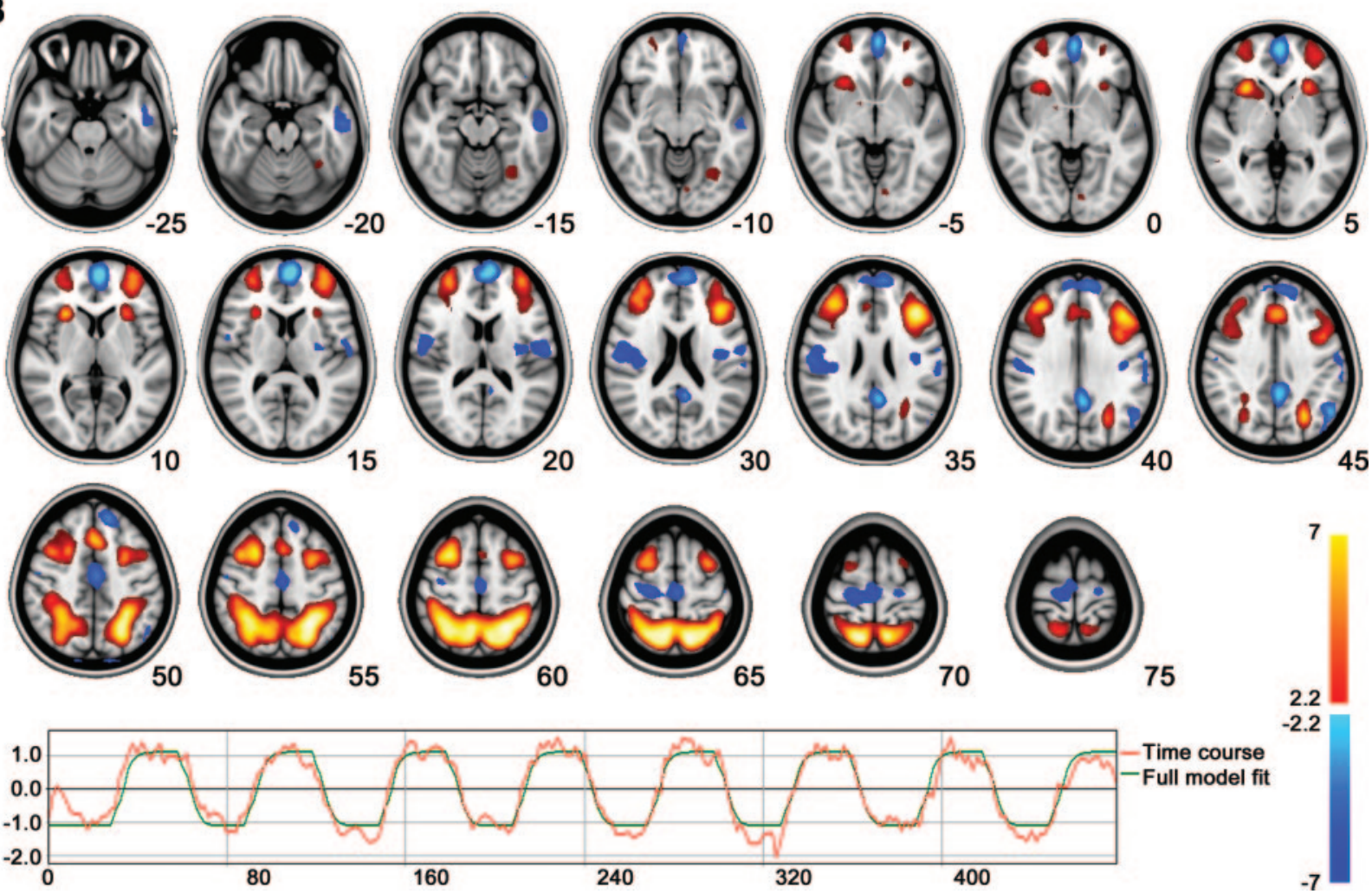

(A) Spatial map of IC1 (0-back >2-back) corresponding to the default mode network and its associated time series. (B) Spatial map of IC2 (2-back $>0$-back) corresponding to working memory network and its associated time series. Hot and cold colors are used to represent activations and deactivations respectively. In the time-series plots, red line represents the independent component time course and green line the task time design. Images are in radiologic convention. 

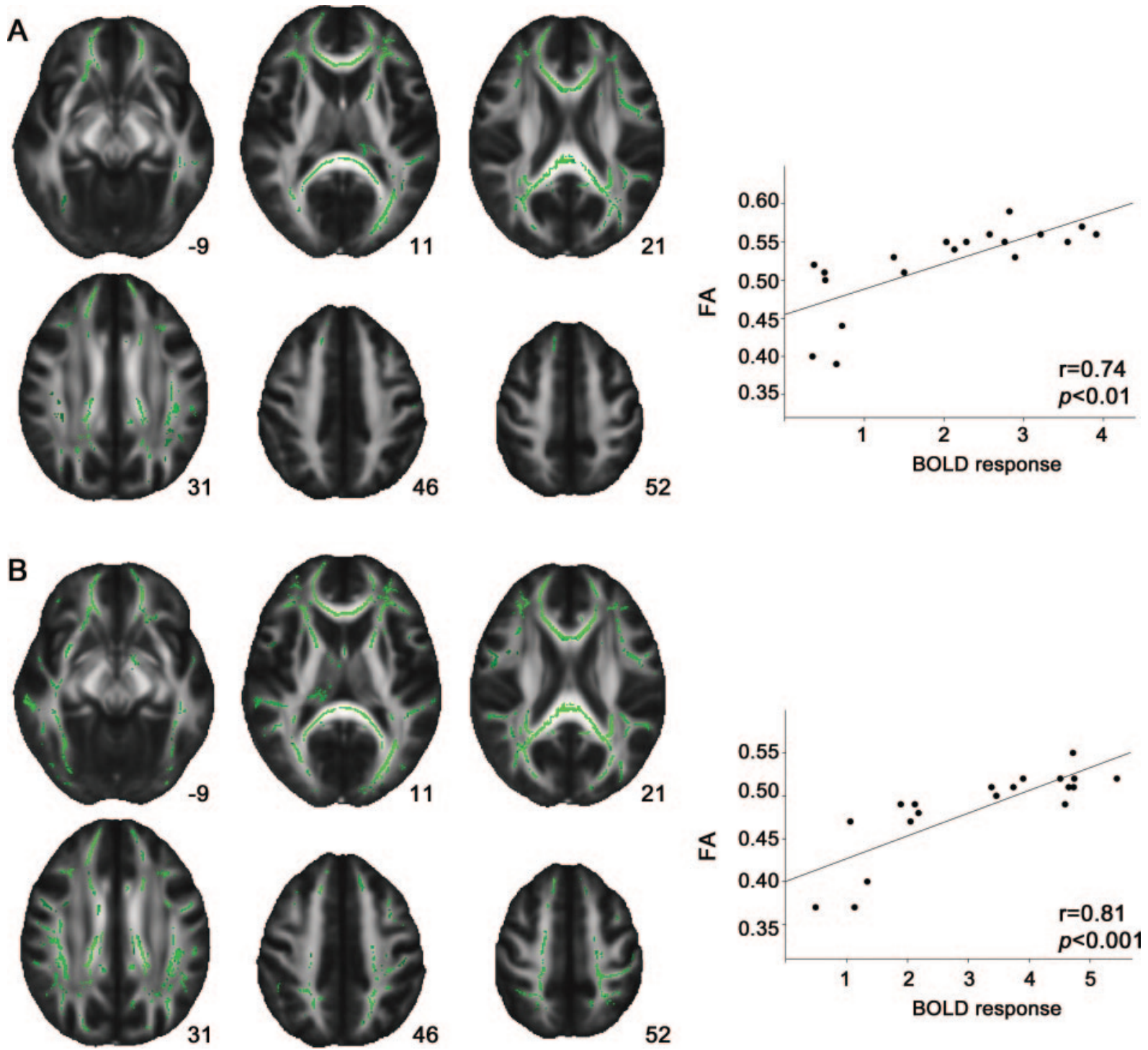

The relationship between fractional anisotropy (FA) values and functional activation scores is represented in green on the standard Montreal Neurological Institute brain (in A for default mode network, and in B for working memory network). Maps are thresholded at $p<0.05$, family-wise error corrected. The fasciculi that achieved significance are described in the main text. Scatterplots show individual values for mean FA within the fasciculi and mean blood oxygenation level-dependent (BOLD) response.

default mode network, which is known to be active during rest and deactivated during externally oriented tasks. ${ }^{21,22}$ The other pattern of activation corresponded to the task-related condition with activation of core areas of working memory circuitry. We observed a relationship between the activation of the working memory network and the deactivation of the default mode network. This finding is in agreement with a previous report which concluded that people who require more cerebral resources to perform a cognitive task will show greater deactivation of the default mode network. ${ }^{23}$ Group comparison showed that patients had decreased brain activity in the default mode network as reported in TBI patients with different severity of injury. ${ }^{24-26}$
We observed widespread patterns of correlation of several white matter fasciculi, the default mode, and working memory networks. Patients with lower fractional anisotropy values had lower brain activation for both default mode and working memory networks. The lack of white matter specificity of these correlations suggests that the decrease in fractional anisotropy values due to the diffuse axonal injury explains the decrease in functional activation. In this case, FA might be reflecting the microbleed effects due to traumatic axonal injury pathology. ${ }^{27}$

When we focused on the working memory performance, only the interhemispheric and intrahemispheric associative fasciculi correlated with working memory function. Whole brain DTI maps revealed 
Figure 3 White matter damage that correlates with altered functional working memory performance
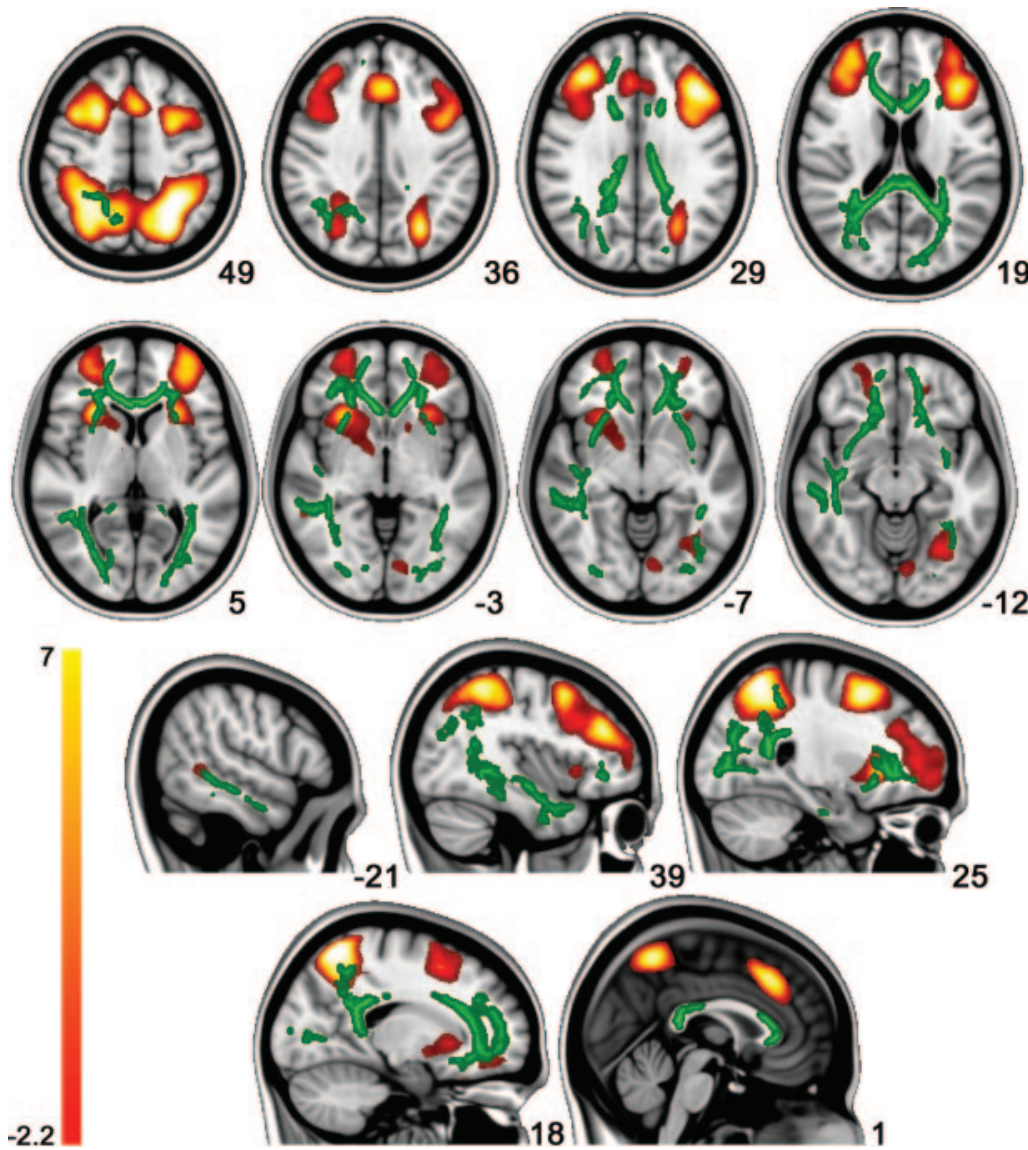

$-12$

$-2.2$

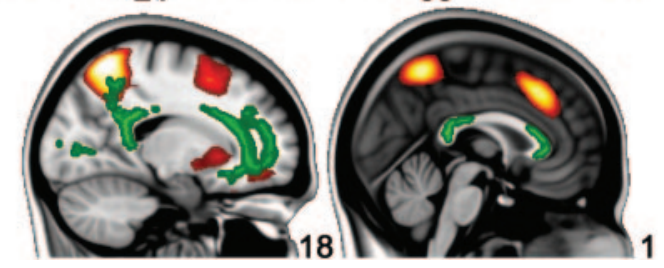

In green, correlations of whole fractional anisotropy values and working memory performance. The fasciculi that achieved significance were posterior part of the superior longitudinal fasciculus (right), inferior longitudinal fasciculi, inferior fronto-occipital fasciculi, and corpus callosum (genu and splenium). Maps are thresholded at $p<0.05$, family-wise error corrected. The activation maps for the working memory task are represented in hot colors. We can see that these significant fasciculi are linking cerebral regions that form part of the working memory network.

that structural damage in specific tracts such as the inferior and superior longitudinal fasciculi, inferior fronto-occipital fasciculi, cingulum, uncinate, and corpus callosum explained the alteration of the lower performance. The superior longitudinal fasciculus is the main fasciculus linking the parietal and frontal lobes and hence it probably plays an important role in working memory. Relations between the superior longitudinal fasciculi and working memory deficits have been reported in multiple sclerosis ${ }^{28}$ and in TBI. ${ }^{9}$ The inferior longitudinal fasciculus also correlated with working memory performance. We expected this finding since in our working memory task the fusiform gyrus activation was recruited and this structure is domain-specific for face recognition. ${ }^{29}$ The structural integrity of corpus callosum also correlated with working memory performance. The corpus callosum is the largest white matter fasciculus and is especially vulnerable after traumatic brain in- jury. ${ }^{30,31}$ As it connects both hemispheres, its damage interrupts the transfer of information that is crucial for efficiency in cognitive performance. . $^{32,33}$ In summary, all these fasciculi link cerebral regions that form part of the working memory network as identified in the fMRI analyses and damage to them is associated with working memory deficits. These results are in agreement with previous reports which found that specific patterns of FA decreases correlated with different cognitive domains such as declarative memory and executive functions. ${ }^{27,34}$ In aged subjects, global FA decreases correlated with speed of mental processing, probably indicating a widespread white matter integrity loss. ${ }^{35}$

We found that patients who performed better had higher activations of the working memory network. Similarly, patients who presented greater deactivation of the default mode network also had better performance. The relationship between the reduction of brain activity in the regions forming part of the default mode network and poorer performance supports the concept that dysfunction in this network is associated with cognitive impairment, as has been reported in other neurologic diseases. ${ }^{36,37}$ Our results indicate that working memory impairment may be a result of a deficit in activated task-related areas together with deficits in deactivating the default mode network during task processing.

The study of the BOLD response in patients with vascular damage, such as severe TBI, may distort attempts to establish the relationship between cognitive impairment and cerebral activation. This is because diffuse vascular brain damage per se may reduce the BOLD response, and this reduction may be mistaken for lower cerebral activation in response to a cognitive task. ${ }^{38}$ We used the activation in the visual network as a control for the working memory task, and we did not observe differences between patients and controls. Therefore our results for hypoactivation during working memory in patients do not seem to be due to a generalized effect of a decreased BOLD response secondary to vascular damage.

The use of an approach that combines different imaging modalities offers an excellent opportunity to elucidate the underlying structural and functional substrates of cognitive deficits. The present study provides strong evidence of the role of structural damage in dysfunctional patterns of working memory and default mode networks in TBI patients. Both structural and functional alterations contribute to working memory deficits.

\section{AUTHOR CONTRIBUTIONS}

E.P., R.S.L.L., and C.J. made substantial contributions to the conception and design of the study, the interpretation of data, as well as to the preparation of the first draft and further revisions of the manuscript. Neuroim- 
aging data were analyzed by E.P. and R.S.L.L. Neuroimaging sequence acquisitions and neuroradiologic evaluations of the MRI were performed by N.B. T.R. participated in the collection of neuropsychological acute clinical data. J.T. and P.V. made a critical revision of the manuscript for important intellectual content, providing additional comments and contributions. C.J. supervised the study. All authors contributed in the discussion and approved the final version of the manuscript.

\section{ACKNOWLEDGMENT}

The authors thank Cesar Garrido from the Centre de Diagnostic per la Imatge of the Hospital Clinic (CDIC) for assistance with data recollection.

\section{DISCLOSURE}

E.M. Palacios was supported by a fellowship from the Institute of Biomedical Research August Pi i Sunyer (IDIBAPS) and research support from the MAPFRE Foundation. R. Sala-Llonch was supported by a fellowship from the Institute of Biomedical Research August Pi i Sunyer (IDIBAPS). Dr. Junque, Dr. Vendrell, Dr. Tormos, Dr. Roig, and Dr. Bargallo report no disclosures.

Received May 18, 2011. Accepted in final form August 16, 2011.

\section{REFERENCES}

1. Johansen-Berg H, Rushworth MF. Using diffusion imaging to study human connectional anatomy. Annu Rev Neurosci 2009;32:75-94.

2. Honey C, Sporns O, Cammoun L, et al. Predicting human resting- state connectivity from structural connectivity. Proc Natl Acad Sci USA 2009;106:2035-2040.

3. Povlishock JT, Katz DI. Update of neuropathology and neurological recovery after traumatic brain injury. J Head Trauma Rehabil 2005;20:76-94.

4. Scheid R, Walther K, Gurthke T, et al. Cognitive sequelae of diffuse axonal injury. Arch Neurol 2006;63:418-424.

5. Christodoulou C, DeLuca J, Ricker JH, et al. Functional magnetic resonance imaging of working memory impairment after traumatic brain injury. J Neurol Neurosurg Psychiatry 2001;71:161-168.

6. Newsome MR, Scheibel RS, Steinberg JL, et al. Working memory brain activation following severe traumatic brain injury. Cortex 2007;43:95-111.

7. Turner GR, Levine B. Augmented neural activity during executive control processing following diffuse axonal injury. Neurology 2008;71:812-818.

8. Sanchez-Carrion R, Gomez PV, Junque C, et al. Frontal hypoactivation on functional magnetic resonance imaging in working memory after severe diffuse traumatic brain injury. J Neurotrauma 2008;25:479-494.

9. Palacios EM, Fernandez-Espejo D, Junque C, et al. Diffusion tensor imaging differences relate to memory deficits in diffuse traumatic brain injury. BMC Neurol 2011;11:24.

10. Biswal BB, Mennes M, Zuo XN, et al. Toward discovery science of human brain function. Proc Natl Acad Sci USA 2010;107:4734-4739.

11. Van den Heuvel MP, Hulshoff Pol HE. Exploring the brain network: A review on resting-state fMRI functional connectivity. Eur Neuropsychopharmacol 2010;20:519_ 534.

12. Warner MA, Youn TS, Davis T, et al. Regionally selective atrophy after traumatic axonal injury. Arch Neurol 2010; 67:1336-1344.

13. Wechsler D. Escala de inteligencia de Wechsler para adultos (WAIS-III). Madrid: TEA Ediciones; 1999.
14. Lezak MD, Howieson DB, Loring DD, et al. Neuropsychological Assessment. New York: Oxford University Press; 2004.

15. Minear M, Park DC. A lifespan database of adult facial stimuli. Behav Res Methods Instruments Computers 2004;36:630-633.

16. Owen AM, McMillan KM, Laird AR, et al. N-back working memory paradigm: a meta-analysis of normative functional neuroimaging studies. Hum Brain Mapp 2005;25: 46-59.

17. Beckmann CF, Smith SM. Probabilistic independent component analysis for functional magnetic resonance imaging. IEEE Trans Med Imaging 2004;23:137-152.

18. Nichols TE, Holmes AP. Nonparametric permutation tests for functional neuroimaging: a primer with examples. Hum Brain Mapp 2002;15:1-25.

19. Hayasaka S, Nichols TE. Validating cluster size inference: random field and permutation methods. Neuroimage 2003;20:2343-2356.

20. Smith SM, Nichols TE. Threshold-free cluster enhancement: addressing problems of smoothing, threshold dependence and localization in cluster inference. Neuroimage 2009;44:83-98.

21. Greicius MD, Krasnow B, Reiss AL, Menon V. Functional connectivity in the resting brain: a network analysis of the default mode hypothesis. Proc Natl Acad Sci USA 2003; 100:253-258.

22. Raichle ME, MacLeod AM, Snyder AZ, et al. A default mode of brain function. Proc Natl Acad Sci USA 2001;98: 676-682.

23. Sumowski JF, Wylie GR, Deluca J, Chiaravalloti N. Intellectual enrichment is linked to cerebral efficiency in multiple sclerosis: functional magnetic resonance imaging evidence for cognitive reserve. Brain 2010;133:362-374.

24. Mayer AR, Mannell MV, Ling J, Gasparovic C, Yeo RA. Functional connectivity in mild traumatic brain injury. Hum Brain Mapp 2011;32:1825-1835.

25. Nakamura T, Hillary FG, Biswal BB. Resting network plasticity following brain injury. PLoS One 2009;4:e8220.

26. Vanhaudenhuyse A, Noirhomme Q, Tshibanda LJ, et al. Default network connectivity reflects the level of consciousness in non-communicative brain-damaged patients. Brain 2010;133:161-171.

27. Kinnunen KM, Greenwood R, Powell JH, et al. White matter damage and cognitive impairment after traumatic brain injury. Brain 2011;134:449-463.

28. Bonzano L, Pardini M, Mancardi GL, Pizzorno M, Roccatagliata L. Structural connectivity influences brain activation during PVSAT in multiple sclerosis. Neuroimage 2009;44:9-15.

29. Kanwisher N. Functional specificity in the human brain: A window into the functional architecture of the mind. Proc Natl Acad Sci USA 2010;107:11163-11170.

30. Levin HS, Williams DH, Valastro M, Eisenberg HM, Crofford MJ, Handel SF. Corpus callosal atrophy following closed head injury: detection with magnetic resonance imaging. J Neurosurg 1990;73:77-78.

31. Kumar R, Saksena S, Husain M, et al. Serial changes in diffusion tensor imaging metrics of corpus callosum in moderate traumatic brain injury patients and their correlation with neuropsychometric tests: a 2-year follow-up study. J Head Trauma Rehabil 2010;25:31-42.

32. Benavidez DA, Fletcher JM, Hannay HJ, et al. Corpus callosum damage and interhemispheric transfer of infor- 
mation following closed head injury in children. Cortex 1999;35:315-336.

33. Wilde EA, Chu Z, Bigler ED, et al. Diffusion tensor imaging in the corpus callosum in children after moderate to severe traumatic brain injury. J Neurotrauma 2006;23:1412-1426.

34. Kraus MF, Susmaras T, Caughlin BP, et al. White matter integrity and cognition in chronic traumatic brain injury: a diffusion tensor imaging study. Brain 2007; 130:2508-2519.

35. Penke L, Muñoz Maniega S, Murray C, et al. A general factor of brain white matter integrity predicts information processing speed in healthy older people. J Neurosci 2010; 30:7569-7574.

36. Greicius MD, Srivastava G, Reiss AL, Menon V. Defaultmode network activity distinguishes Alzheimer's disease from healthy aging: evidence from functional MRI. Proc Natl Acad Sci USA 2004;101:4637-4642.

37. Rocca MA, Valsasina P, Absinta M, et al. Default-mode network dysfunction and cognitive impairment in progressive MS. Neurology 2010;74:1252-1259.

38. Hillary FG, Biswal B. The influence of neuropathology on the fMRI signal: a measurement of brain or vein? Clin Neuropsychol 2007;21:58-72. 